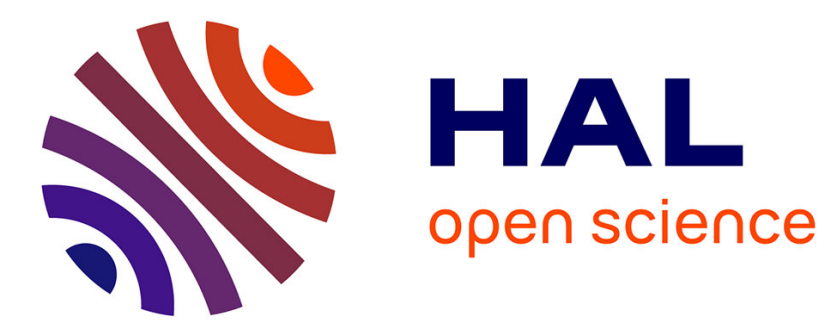

\title{
Magnetic properties, anisotropy parameters and magnetocaloric effect of flux grown MnFe4Si3 single crystal
}

H. Yibole, W. Hanggai, Z.Q. Ou, Radia Hamane, V Hardy, François Guillou

\section{- To cite this version:}

H. Yibole, W. Hanggai, Z.Q. Ou, Radia Hamane, V Hardy, et al.. Magnetic properties, anisotropy parameters and magnetocaloric effect of flux grown MnFe4Si3 single crystal. Journal of Magnetism and Magnetic Materials, 2020, 504, pp.166597. 10.1016/j.jmmm.2020.166597 . hal-03022338

\section{HAL Id: hal-03022338 \\ https://hal.science/hal-03022338}

Submitted on 24 Nov 2020

HAL is a multi-disciplinary open access archive for the deposit and dissemination of scientific research documents, whether they are published or not. The documents may come from teaching and research institutions in France or abroad, or from public or private research centers.
L'archive ouverte pluridisciplinaire HAL, est destinée au dépôt et à la diffusion de documents scientifiques de niveau recherche, publiés ou non, émanant des établissements d'enseignement et de recherche français ou étrangers, des laboratoires publics ou privés. 


\title{
Magnetic properties, anisotropy parameters and magnetocaloric effect of flux grown $\mathrm{MnFe}_{4} \mathrm{Si}_{3}$ single crystal
}

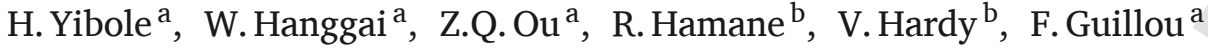 \\ a Inner Mongolia Key Laboratory for Physics and Chemistry of Functional Materials, Inner Mongolia Normal University, 81 Zhaowuda $R^{d}$, Saihan District, Hohhot 010022, China \\ ${ }^{\mathrm{b}}$ Laboratoire CRISMAT, UMR 6508, CNRS/ENSICAEN/UCBN, $6 B^{d}$ Marechal Juin, 14050 Caen Cedex, France
}

\section{A R T I C L E I N F O}

\section{Keywords}

Single-crystal growth

Magnetic anisotropy

Magnetocaloric effect

Transition-metal alloys

\begin{abstract}
A B S T R A C T
The transition-metal based alloy $\mathrm{MnFe}_{4} \mathrm{Si}_{3}$ not only is a potential candidate for room temperature magnetocaloric applications, but also shows a large magnetic anisotropy forming an interesting case study in the search for rare-earth free permanent magnets. However, former polycrystalline and single crystal studies led to major disagreements about the order of the magnetic transition and the magnetocrystalline anisotropy scheme, which are two essential points for the understanding of this alloy. Here, magnetic, magnetocaloric properties and the magnetic anisotropy of $\mathrm{MnFe}_{4} \mathrm{Si}_{3}\left(\mathrm{Mn}_{\sim 0.86} \mathrm{Fe}_{\sim 4.24} \mathrm{Si}_{\sim 2.90}\right)$ are investigated on a high quality single crystal grown by flux method, and compared to polycrystalline materials. Using the recently proposed criterion of field dependence of the magnetocaloric effect, we show that the ferromagnetic transition is more likely to be of second order, which is fully compatible with the absence of thermal hysteresis at the ferromagnetic transition in the present $\mathrm{MnFe}_{4} \mathrm{Si}_{3}$ crystal. The $c$ axis is confirmed to be the hard magnetic axis, both in single crystal and polycrystalline $\mathrm{MnFe}_{4} \mathrm{Si}_{3}$, and a large, dominant, $K_{1}$ anisotropy constant $\left(\sim-2.5 \mathrm{MJ} \mathrm{m}^{-3}\right)$ is found at low temperatures.
\end{abstract}

\section{Introduction}

Initially investigated in the $60 \mathrm{~s}$ and $70 \mathrm{~s}$ [1-4], transition metal-based compounds deriving from $\mathrm{Mn}_{5-x} \mathrm{Fe}_{\mathrm{X}} \mathrm{Si}_{3}$ are currently receiving a renewed interest for their rich magnetic properties and for their potential use in magnetocaloric applications [5-11]. $\mathrm{Mn}_{5-x} \mathrm{Fe}_{\mathrm{x}} \mathrm{Si}_{3}$ compounds were originally believed to crystallize in the hexagonal $P_{6_{3} / \mathrm{mcm}}$ structure with $\mathrm{Mn}$ and Fe distributed on $4 d$ and $6 g$ sites, and Si metalloid atoms on $6 g$ sites with different coordinates. Magnetic properties were found to be highly dependent on the Fe content $[5,6]$. Of particular interest is the $x \approx 4$ composition, $\mathrm{MnFe}_{4} \mathrm{Si}_{3}$, which is ferromagnetic with a saturation magnetization of 1.5-1.7 $\mu_{\mathrm{B}} /$ T.M. and presents a Curie temperature around $300 \mathrm{~K}$ making it a potential candidate for magnetocaloric applications near room temperature [5]. Some recent studies have deepened the understanding of this materials family using single crystals grown by Czochralski method [8]. Combined neutron and X-ray diffraction data have led to the emergence of a new structural model [8] and detailed inelastic neutron scattering experiments and Mössbauer spectroscopy have clarified the spin and lattice dynamics of $\mathrm{MnFe}_{4} \mathrm{Si}_{3}[9,10]$.

Despite these extensive investigations on $\mathrm{MnFe}_{4} \mathrm{Si}_{3}$, some controversies persist. First, the order of the magnetic transition $\mathrm{MnFe}_{4} \mathrm{Si}_{3}$ is unclear. The ferromagnetic transition was originally classified as a contin- uous second order transition, due to the absence of field-induced transition on the magnetization curves of bulk polycrystalline materials [5]. However, the recent observation of a large thermal hysteresis of $\sim 10 \mathrm{~K}$ in single crystal is characteristic of a first order transition [8]. Further physical properties pointed out a complex behavior potentially closer to a second order behavior [9]. Usually, observing a large thermal hysteresis at a magnetic transition is typical of a first order transition with a significant latent heat and large discontinuities in the lattice parameters or a change in crystal symmetry. All these features should in turn lead to appreciable magnetocaloric entropy change [12]. On the contrary, $\mathrm{MnFe}_{4} \mathrm{Si}_{3}$ seems to combine a relatively large thermal hysteresis with only a modest entropy change of $2-2.5 \mathrm{~J} \mathrm{~kg}^{-1} \mathrm{~K}^{-1}$ for $2 \mathrm{~T}$, making it an unusual magnetocaloric compound $[5,8,9]$. The second debated issue in $\mathrm{MnFe}_{4} \mathrm{Si}_{3}$ concerns the magnetic anisotropy, originally believed to correspond to a $c$ easy axis from neutron diffraction measurements and X-ray diffraction on in-field oriented powders [3], but later proposed to be a $c$ hard axis with an easy $(a, b)$ plane based on single crystal data [8].

The purpose of this work is to clarify the two aforementioned points, by a detailed study of the order of the transition from magnetization and magnetocaloric measurements, and by investigating the magnetic anisotropy of $\mathrm{MnFe}_{4} \mathrm{Si}_{3}$ both in single crystal and polycrystalline forms. A single crystal growth technique different from Ref. [8]

E-mail address: francoisguillou@imnu.edu.cn (F. Guillou) 
was selected for this study in order to investigate whether sample preparation can explain part of the disagreements in the literature. In addition, a quantitative estimate of the magnetic anisotropy parameters over a large temperature range turns out to be needed for $\mathrm{MnFe}_{4} \mathrm{Si}_{3}$, since other 5:3 transition-metal:metalloid alloys such as $\mathrm{Fe}_{5}(\mathrm{Si}, \mathrm{B})_{3}$ or $\mathrm{Fe}_{5}(\mathrm{P}, \mathrm{B})_{3}$ are currently explored for their potential use as rare-earth free permanent magnets [13-15].

\section{Experimental details}

$\mathrm{MnFe}_{4} \mathrm{Si}_{3}$ single crystals were grown by tin flux method. High-purity Mn (99.9\%), Fe (99.99\%) and Si (99.999\%) starting materials were arc-melted with Sn (99.999\%) in Ar atmosphere in a water-cooled copper crucible. Due to the smaller solubility of Si in molten Sn relative to $\mathrm{Mn}$ and $\mathrm{Fe}$, we used extra amount of Si starting materials compared to the metal:metalloid stoichiometric ratio of 5:3. The resulting ingot was then sealed in quartz ampoule in 200 mbar Ar. The charge to flux ratio was $1: 20 \mathrm{wt} \%$. The sealed ampoule was then placed in a vertical furnace and heated up to $1423 \mathrm{~K}$. The sample was maintained at this temperature for $100 \mathrm{~h}$, and then slowly cooled at a rate of $2 \mathrm{~K} / \mathrm{h}$ down to $700 \mathrm{~K}$, at which temperature the excess of $\mathrm{Sn}$ was removed. The remaining flux was finally removed by etching with diluted hydrochloric acid. The as-grown crystals present the well-formed prismatic shape of an elongated needle. Microanalyses were performed using Hitachi SU-8010 scanning electron microscope (SEM) equipped with an energy dispersive spectrometer (EDS). The chemical compositions of the single crystals were determined with EDS by probing several locations on each crystal at an accelerating voltage of $15 \mathrm{keV}$. Reference materials were used to correct EDS data and make the chemical analysis more quantitative.

A polycrystalline $\mathrm{MnFe}_{4} \mathrm{Si}_{3}$ ingot was prepared by arc-melting elemental starting materials in purified Ar atmosphere. The button was flipped 6 times to ensure homogenization. The grain size distribution of this as-prepared button is quite large, with a few crystallites at the button surface having an elongated shape and reaching the $\mathrm{mm}$ in length, while the inner part of the button present a columnar microstructure with an average grain size of approximately $120 \mu \mathrm{m} \times 40 \mu \mathrm{m}$. The resulting sample is then cut, ground using an agate mortar and sieved. Particles smaller than $36 \mu \mathrm{m}$ are used for powder x-ray diffraction, and part of the powder is oriented in a magnetic field of $11 \mathrm{kOe}$ using epoxy glue as binder. Powder X-ray diffraction XRD experiments were carried out at room temperature using a Panalytical Empyrean diffractometer employing $\mathrm{Cu} \mathrm{K}_{\alpha}$ radiation.

Magnetic measurements were performed in a VersaLab Quantum Design system employing a Vibrating Sample Magnetometer option. To limit possible thermalization artefacts, magnetization measurements as a function of the temperature were carried out while settling the temperature at each point and/or using slow temperature sweep $\left(<1 \mathrm{~K} \mathrm{~min}^{-1}\right)$. The limited size of the sample ( $m \sim 0.019 \mathrm{mg}$, estimated from SEM pictures and the theoretical density of $\mathrm{MnFe}_{4} \mathrm{Si}_{3}$ ) makes such measurements challenging. First, to avoid contamination, the needle is mounted under optical microscope on a fresh half-straw quartz holder using nonmagnetic tools and only a tiny amount of superglue as fixer. A background signal with only the glue was measured to ensure it results only in a minor diamagnetic contribution. Then, large oscillations $(4 \mathrm{~mm})$ and long integration times (up to $10 \mathrm{~s}$ /point) were used to optimize the signal/noise ratio. Even in the most unfavorable situation, such as $M-T$ curve recorded along the hard axis with an external field of 100 Oe, the signal in the paramagnetic phase $\left(9.10^{-6} \mathrm{emu}\right)$ remains significantly larger than the noise taken as the scattering between different points in the same conditions (noise level $\sim 2 \cdot 10^{-7} \mathrm{emu}$ ). Neglecting potential error on the density, most of the uncertainty on the massic or volumic magnetization actually originates from the determination of the radius of the needle on SEM pictures, $\Delta r / r \approx 4 \%$, which leads to sizable uncertainty on the sample volume $\Delta V / V \approx 8 \%$ and thus on the normalized magnetization, estimated as $\Delta M / M \approx \Delta m_{\text {raw }} / m_{\text {raw }}+\Delta V / V \approx 10 \%$. The magnetization versus temperature curves are presented in terms of external magnetic field. For $M$ vs $H$ measurements with $H / /(a, b)$ plane, i.e. for a magnetic field applied perpendicular to the long axis of the needle, a demagnetization factor of $N=1 / 2$ was accounted for in order to present $M$ - $H$ data in terms of internal magnetic field $H_{\text {in }}$, according to the usual approximation $H_{\text {in }} \approx H-N M$, where $H$ represents the applied magnetic field.

\section{Results and discussion}

Fig. 1(a) shows an SEM image of the as-grown crystal used in this study. The surfaces of the crystal are regular and homogeneous without noticeable flux contamination, and the needle shape presents a length: diameter ratio more than 30:1. The composition calculated from EDS spectrometry is approximately $\mathrm{Mn}_{\sim 0.86} \mathrm{Fe}_{\sim 4.24} \mathrm{Si}_{\sim 2.90}$, i.e. close to the targeted composition but with possibly a slight excess of Fe. For simplicity only the $\mathrm{MnFe}_{4} \mathrm{Si}_{3}$ nominal composition is mentioned hereafter. Fig. 1(b) and (c) show magnetization curves for the single crystal measured along different directions. In the direction perpendicular to the $c$ axis, i.e. perpendicular to the long axis of the needle, the only distinguishable feature is the magnetization step corresponding to the ferromagnetic transition at $323 \mathrm{~K}$ taken from the $\mathrm{d} M / \mathrm{d} T$ maximum in an external field of $1 \mathrm{kOe}$. This transition temperature is slightly higher than the range 300-310 K reported in former studies on $\mathrm{MnFe}_{4} \mathrm{Si}_{3}$, a feature which can be ascribed to the small deviation in stoichiometry inherent to our flux crystal growth method. As a matter of fact, observing $T_{\mathrm{C}}$ slightly higher than $300-310 \mathrm{~K}$ is fully compatible with a minor Fe excess $(x \geq 4)$ in the $\mathrm{Mn}_{5-x} \mathrm{Fe}_{x} \mathrm{Si}_{3}$ phase diagram [6]. While no significant magnetic anisotropy could be distinguished when measuring magnetization along 3 random directions with $H \perp c$ (not shown), the shape of the $M(T)$ curve is strongly modified and the magnetization strongly reduced when performing measurements in $H / / \mathrm{c}$ direction. This indicates a combination of an easy $(a, b)$ plane with an hard $c$ axis uniaxial magnetic anisotropy, in line with recent single crystal investigation [8]. However in contrast to a former study reporting a $10 \mathrm{~K}$ thermal hysteresis [8], our data shows that the shift between heating and cooling $M(T)$ curves is smaller than $0.1 \mathrm{~K}$ (both for measurements parallel or perpendicular to the $c$ axis), pointing to the absence of significant thermal hysteresis at the ferromagnetic transition in the present crystal. On the one hand, the presence of thermal hysteresis at a magnetic transition can be regarded as a signature of its first-order nature. On the other hand, absence of hysteresis does not demonstrate one is dealing with a second-order transition, since there are first-order magnetic transitions with very small thermal hysteresis [16].

To get further insight into the ferromagnetic transition order in $\mathrm{MnFe}_{4} \mathrm{Si}_{3}$, we used the method recently proposed by V. Franco et al. which consists in analyzing the temperature and field dependence of the magnetocaloric effect $[17,18]$. It requires to first determine the magnetocaloric $\Delta S$, as presented in Fig. 2 in the easy plane direction, which is the most relevant for caloric purposes. Given that no significant thermal hysteresis could be detected, the entropy change was calculated based on magnetization versus field curves measured at different temperatures and using the magnetocaloric Maxwell equation [19]. Finite entropy change values are found over a broad temperature range around the Curie temperature. The maximal $\Delta S$ are $-1.8,-2.9$ and $-3.7 \mathrm{~J} \mathrm{~kg}^{-1} \mathrm{~K}^{-1}$ for field changes $\Delta B=1,2$ and $3 \mathrm{~T}$, respectively. These entropy change values are -as expected- larger than the $\sim 2 \mathrm{Jkg}^{-1} \mathrm{~K}^{-1}$ for $\Delta B=2 \mathrm{~T}$ reported in polycrystalline materials [5], and in line with $2-2.9 \mathrm{Jkg}^{-1} \mathrm{~K}^{-1}$ reported for single crystals $[8,9]$ or calculated by Monte Carlo simulations [11]. While sizable, these entropy values are nevertheless relatively limited, as the magnetocaloric effect spreads over a large temperature range, mimicking the broadening of $M(T)$ curves at high field. Such a behavior is most often typical of magnetocaloric materials with second order continuous transitions. 
(a)

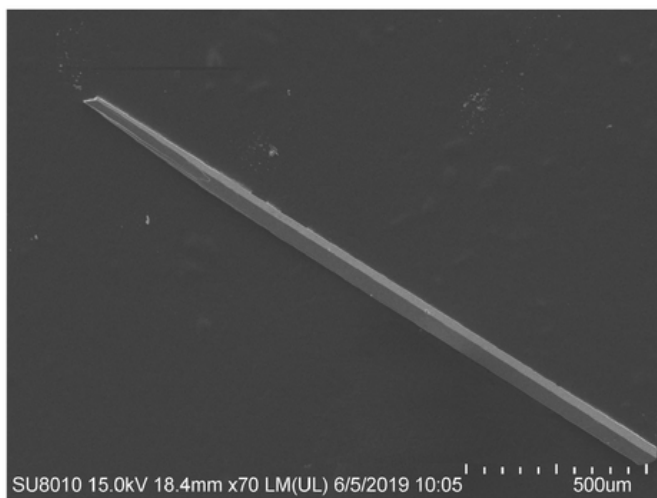

(b)

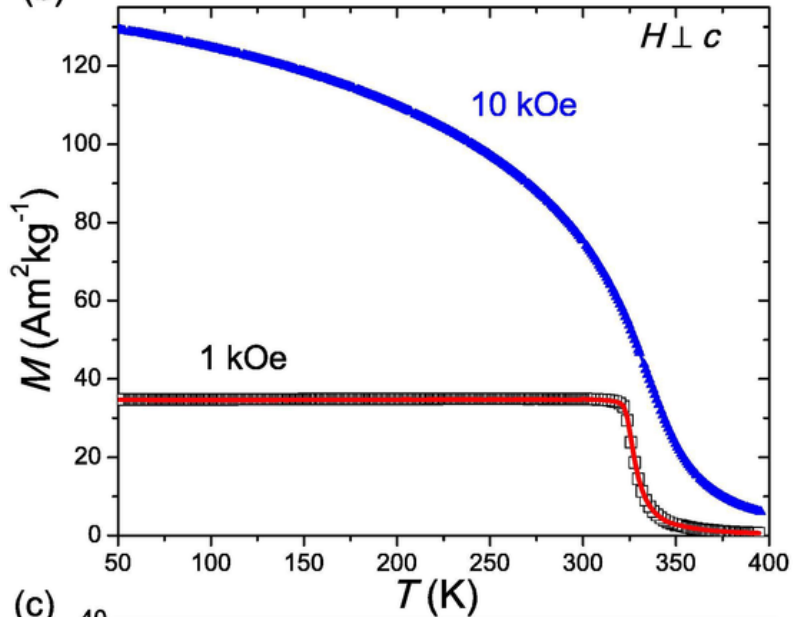

(c)

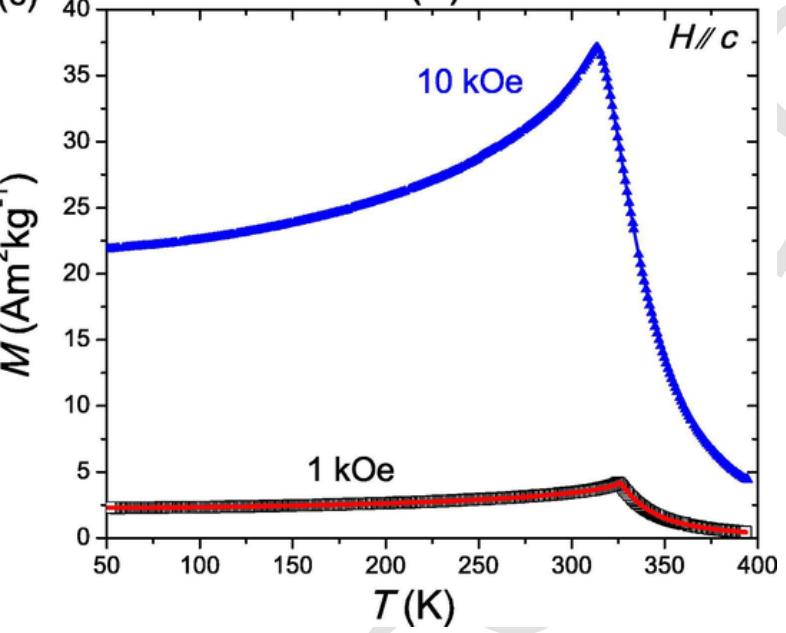

Fig. 1. SEM picture of $\mathrm{MnFe}_{4} \mathrm{Si}_{3}$ crystal used in this study (a) and magnetization measurements as a function of the temperature in an external field of 1 kOe upon cooling (open symbol) and upon heating (line) and $10 \mathrm{kOe}$ perpendicular to the long axis (b) and parallel (c).

Then, assuming that the field-dependence of the isothermal entropy change obeys a power law $\left(\Delta S \propto H^{n}\right)$, one calculates the exponent $\mathrm{n}$ from the expression: $n(T, H)=d \ln |\Delta S| / d \ln H$ [18]. Several studies have shown that the field dependence of the magnetocaloric effect is intrinsically different depending on the order of the magnetic transition $[17,18,20]$. In particular, it has recently been proposed that $n>2$ in the transition region is characteristic of first order magnetic transition [18]. Bottom panel of Fig. 2 presents the local field dependence of $\Delta S$ in $\mathrm{MnFe}_{4} \mathrm{Si}_{3}$ single crystal. Clearly, $n$ remains lower than 2 near $T_{\mathrm{C}}$ for $\Delta B=2 \mathrm{~T}$, and similar results were obtained for other field change val-
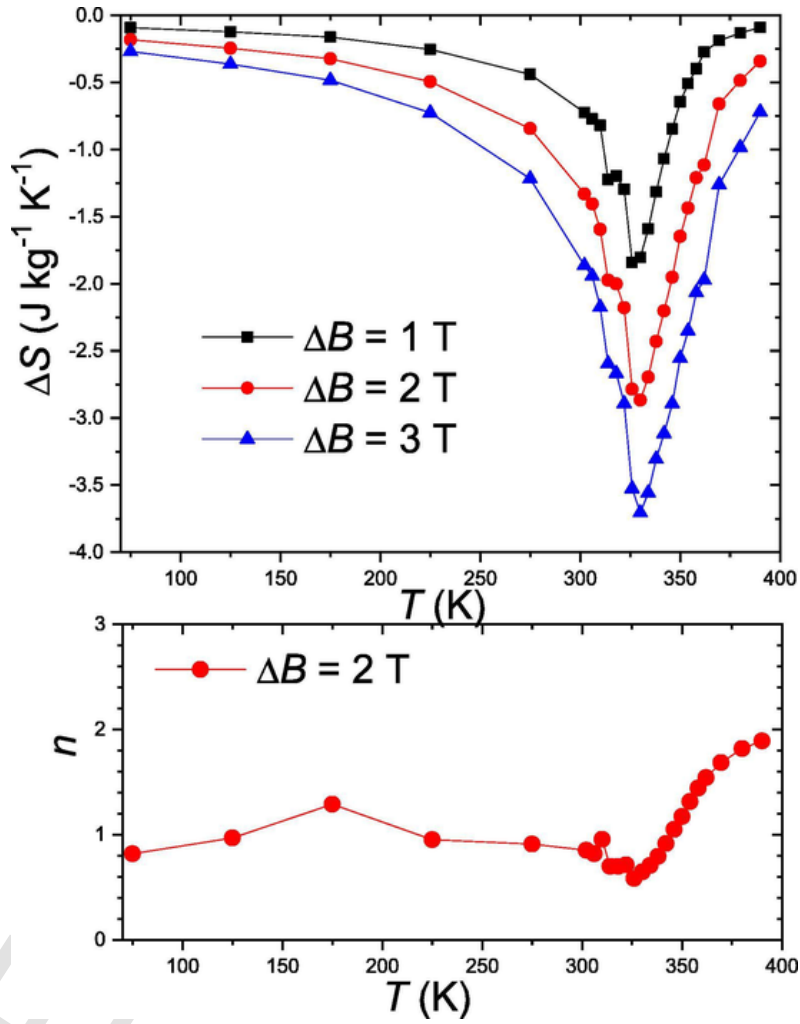

Fig. 2. Entropy change as a function of the temperature for $\mathrm{MnFe}_{4} \mathrm{Si}_{3}$ crystal in the easy plane direction. Bottom panel, local field dependence of the entropy change at $2 \mathrm{~T}$.

ues (not shown). Not only the $n$ values, but also their temperature evolution, $\sim 1 \rightarrow$ minimum $\rightarrow \sim 2$, are typical of materials with second order transition [18]. When $T \gg T_{\mathrm{C}}, n$ tends toward 2, the values expected for a conventional paramagnet. The analysis of the field dependence of the $\Delta S$ thus demonstrates the second order character of the ferromagnetic transition, in line with the relative broadness of transition at intermediate magnetic fields and the absence of thermal hysteresis on the $M(T)$ curves on this crystal.

Another point of controversy that emerged from past studies concerns the magnetic configuration and magnetic anisotropy. The first powder neutron diffractions studies reported for $\mathrm{MnFe}_{4} \mathrm{Si}_{3}$ spin arrangement with moments tilted by $\sim 40^{\circ}$ of the $c$ axis [6] or oriented along the $c$ axis [7], in a way similar to the parent $\mathrm{Fe}_{5} \mathrm{Si}_{3}$ [2]. On the other hand, more recent studies based on single crystals revealed a spin arrangement with moment in the $(a, b)$ plane from magnetization measurements and at $136^{\circ}$ to $\left[\begin{array}{lll}1 & 0 & 0\end{array}\right]$-direction from neutron diffraction [8]. Magnetization versus field curves were systematically recorded on our single crystal in the $(a, b)$ plane and along the $c$ axis, a selection of the most representative temperatures are presented in Fig. 3. Only the first quadrant is shown, but the measurements were performed along 5 quadrants and pointed toward a negligibly small magnetic hysteresis. For instance, at $50 \mathrm{~K}$, the coercive field $H_{\mathrm{C}}$ is less than $5 \mathrm{Oe}$ and remnant magnetization $M_{\text {rem }}$ less than $0.05 \mathrm{Am}^{2} \mathrm{~kg}^{-1}$. In agreement with temperature dependence of the magnetization in Fig. 1, $M(H)$ data indicate an $(a, b)$ easy plane with a $c$ hard axis anisotropy. At $50 \mathrm{~K}$, the magnetic anisotropy appears particularly large, as the anisotropy field $\left(H_{\text {an }}\right)$ is significantly higher than our applied field of $3 \mathrm{~T}$. A graphical linear extrapolation suggests that $H_{\text {an }}$ is of the order of $\sim 6 \mathrm{~T}$. This anisotropy slowly decreases while increasing the temperature until approaching the Curie temperature $T_{\mathrm{C}} \approx 323 \mathrm{~K}$ where it decreases more rapidly. Around $300 \mathrm{~K}$, the magnetization parallel and perpendicular to the $c$ axis does not coincide, which points toward a certain anisotropy of the magnetization and the presence of non-zero orbital moments in 
(a)

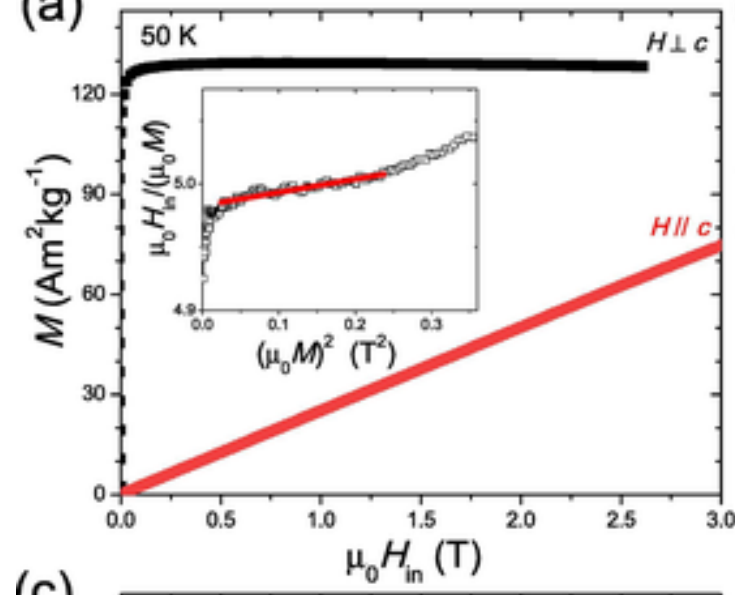

(c)

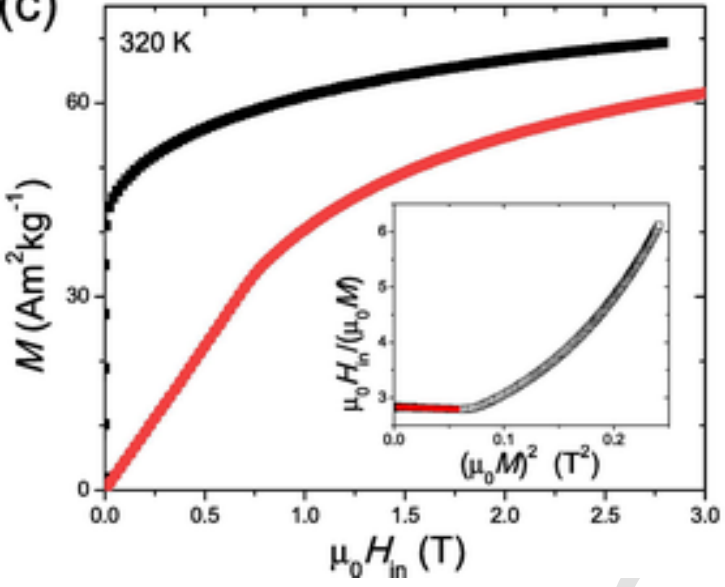

(b)

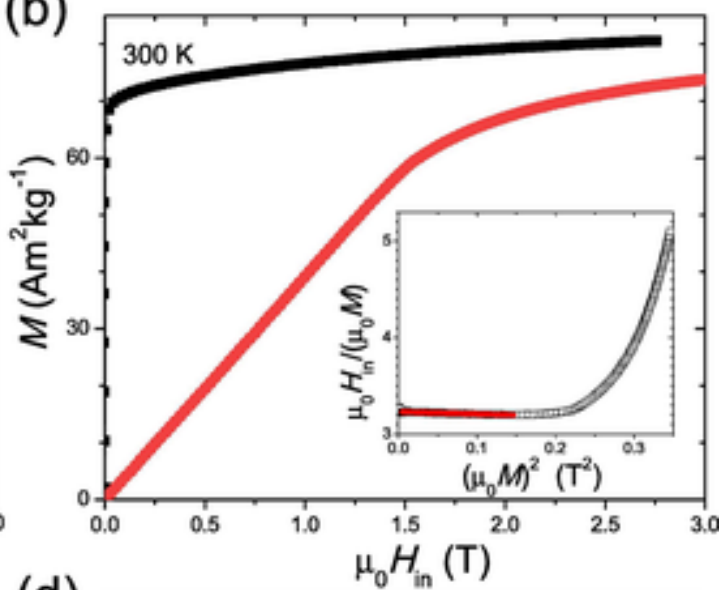

(d)

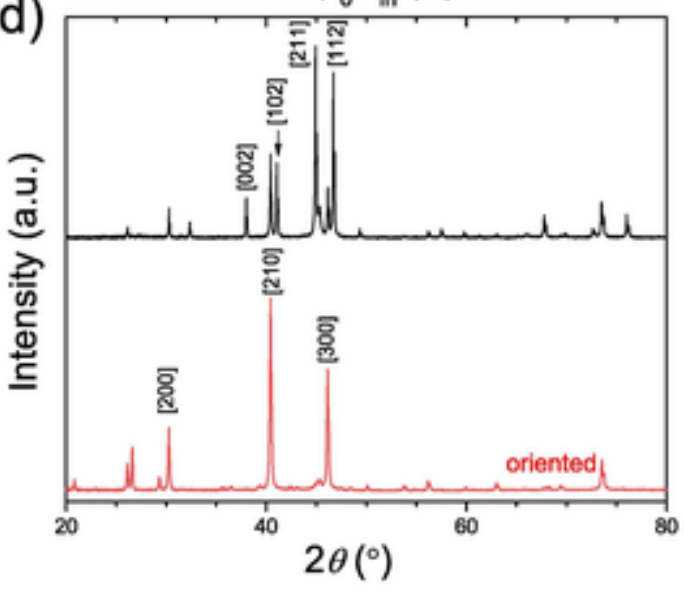

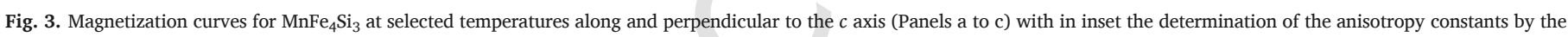
Sucksmith and Thompson method. Panel d, comparison between random (top curve) and oriented (bottom curve) powder XRD with peak experiencing large changes being indexed.

$\mathrm{MnFe}_{4} \mathrm{Si}_{3}$. These observations, in particular the $c$ axis being the hard magnetic axis, are in line with formerly reported magnetization curves on single crystals [8] and at odd with polycrystalline studies [3,6,7]. As the cooling rate during single crystal synthesis is much slower than polycrystalline materials prepared by induction melting [7] or arc-melting with annealing followed by quenching [6], preferential site occupation maybe different in materials with different crystalline forms. To investigate this possibility we prepared a polycrystalline batch of $\mathrm{MnFe}_{4} \mathrm{Si}_{3}$ by arc-melting. Then, the sample is hand crushed into particles and part of this powder is mixed with an epoxy binder, shaped into a plate, and oriented in magnetic field with the orientation field perpendicular to the plate. XRD measurements were made on the plate surface. XRD measurements for random and oriented powders show a stark contrast. As a result of magnetic orientation, one observes an almost complete disappearance of the reflections having a $c$ axis component like for instance the $\left[\begin{array}{lll}0 & 0 & 2\end{array}\right]$ direction; while Bragg peaks having an $a$ or $b$ axis compo-

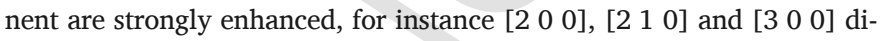
rections. These results appear opposite to that obtained for $\mathrm{Fe}_{5} \mathrm{Si}_{3}$ and $\mathrm{MnFe}_{4} \mathrm{Si}_{3}$ by a similar method, which were indicating an easy axis along the $c$ axis for both alloys [3]. Our powder oriented polycrystalline sample indicates that in polycrystalline materials the $(a, b)$ plane is an easy plane and $c$ is the hard axis, in agreement with single crystal magnetization data. Different crystalline forms can thus not explain the discrepancy in magnetic anisotropy reported for $\mathrm{MnFe}_{4} \mathrm{Si}_{3}$.

The large magnetic anisotropy of $\mathrm{MnFe}_{4} \mathrm{Si}_{3}$ turns out to deserve a more quantitative determination, in particular as transition metal-based alloys could be considered as potential candidates as rare-earth free permanent magnets. Usually for an hexagonal system, the first $\left(K_{1}\right)$ and second-order $\left(K_{2}\right)$ anisotropy constants are considered for the magnetocrystalline anisotropy energy $E \approx K_{1} \sin ^{2} \theta+K_{2} \sin ^{4} \theta$. To determine $K_{1}$ and $K_{2}$ from magnetization measurements along the hard $c$ axis direction, the Sucksmith and Thompson method has been used [21]. This method is more customarily employed to derive the two magneto-crystalline anisotropy constants of materials with $c$ being the easy magnetic axis. It can nevertheless be applied to the case of an easy magnetization direction perpendicular to the hexagonal axis. In such a case, the slope and intercept of an $H / M v s M^{2}$ plot will correspond to slope $=4 K_{2} M_{S}^{4}$ and intercept $=\left(-2 K_{1}-4 K_{2}\right) / M_{S}^{2}$, respectively [21]. The applicability of the Sucksmith and Thompson method to various magneto-crystalline anisotropy scheme has been confirmed from modelling studies [22] and used in different materials with a combination of easy plane and hard $c$ axis $[23,24]$. Beside systematic error, due to the significant relative error on magnetization, the differences between maxima and minima for the intercepts and slopes of $H_{\mathrm{in}} / M v s\left(\mu_{0} M\right)^{2}$ linear fits are large too, resulting in sizable uncertainties on $K_{1}$ and $K_{2}$. Fig. 4 presents the temperature evolution of $K_{1}$ and $K_{2} . K_{2}$ appears negligible compared to the large $K_{1}$. While $K_{1}$ exhibits negative values, $K_{2}$ might experience a change of sign around $150 \mathrm{~K}$. The predominance of $K_{1}$ and its negative sign is in line with an easy plane magnetic direction perpendicular to the $c$ axis [25]. Neglecting $K_{2}$, our estimate of the anisotropy field ( $H_{\text {an }} \sim 6 \mathrm{~T}$ at $T=50 \mathrm{~K}$ ) provides us a mean for an alternative estimation of $K_{1}$ considering $M=M_{\mathrm{S}}$ at $H_{\mathrm{an}}$, it results in $K_{1} \approx-1 / 2$ $\left(H_{\mathrm{an}} M_{\mathrm{S}}\right) \approx-3.0 \mathrm{MJm}^{-3}$. This estimate of $K_{1}$ is fully compatible with that obtained by $H / M v s M^{2}$ fittings $\left(K_{1} \approx-2.8 \mathrm{MJ} \mathrm{m}^{-3}\right.$ ). 


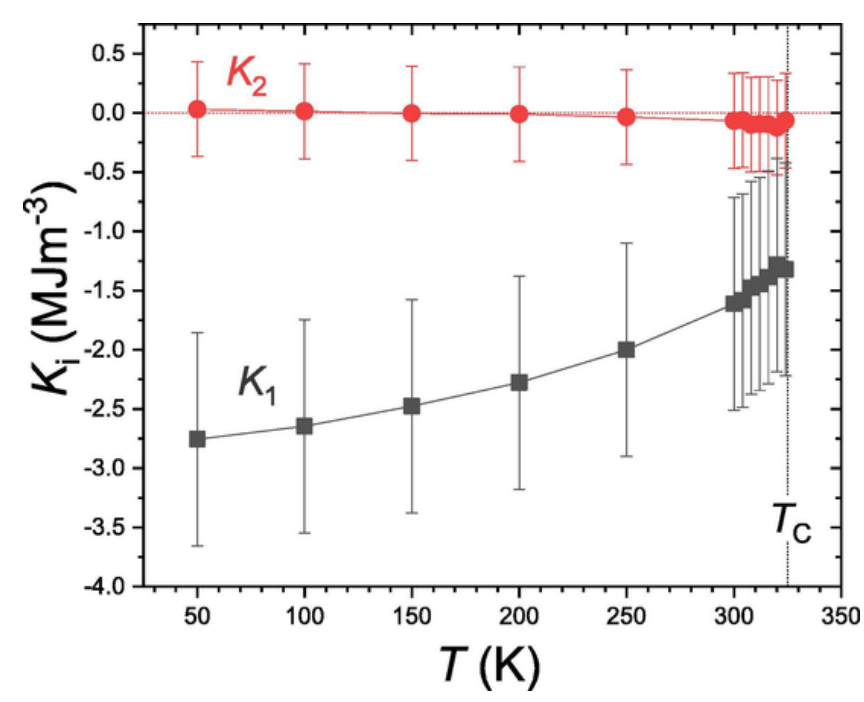

Fig. 4. Temperature dependence of the magneto-crystalline anisotropy parameters for $\mathrm{MnFe}_{4} \mathrm{Si}_{3}$.

The binary parent $\mathrm{Fe}_{5} \mathrm{Si}_{3}$ is ferromagnetic with a $T_{\mathrm{C}} \sim 380 \mathrm{~K}$, but its magnetic anisotropy could unfortunately not be compared to $\mathrm{MnFe}_{4} \mathrm{Si}_{3}$, as being a metastable phase at room temperature stabilized by rapid solidification or nanostructuration, little single crystal information could be found. On the other hand, ternaries with metalloid substitutions on $\mathrm{Si}$ have recently received a great interest as they offer a possibility to reach Curie temperatures higher than those of the binary parents, making them potential candidates for rare-earth free permanent magnet. In tetragonal $\mathrm{Fe}_{5} \mathrm{SiB}_{2}$, between $T_{\mathrm{C}} \sim 784 \mathrm{~K}$ and $140 \mathrm{~K}$ (spin re-orientation transition) the easy magnetic axis is along the $c$ axis with an anisotropy $K_{1}=0.25 \mathrm{MJ} \mathrm{m}^{-3}$, and an easy plane anisotropy below $140 \mathrm{~K}$ with only a modest anisotropy (MAE $=-0.28 \mathrm{MJ} \mathrm{m}^{-3}$ ) $[13,15]$. In this latter tetragonal compounds, $\mathrm{P}$ for $\mathrm{Si}$ substitution favors an easy axis magnetization with magnetic anisotropy $K_{1} \sim 0.38 \mathrm{MJ} \mathrm{m}^{-3}$ at room temperature $[13,14]$. In hexagonal ternary alloys deriving from $\mathrm{Fe}_{5} \mathrm{Si}_{3}$ such as $\mathrm{MnFe}_{4} \mathrm{Si}_{3}$, a different configuration is observed with a relatively strong hard $c$ axis anisotropy and an easy plane. Beside the difference in crystal structure, the incorporation of $\mathrm{Mn}$ in $\mathrm{Fe}_{5} \mathrm{Si}_{3}$ may also play a role in the strengthening of the magnetic anisotropy. Mn atoms carry a significantly larger overall magnetic moment than Fe $[6,7]$, and may also favor a stronger spin-orbit coupling. In addition, spin-polarized calculations pointed out the important role played by Mn $d$ states in the filling of the electronic structure near the Fermi level [7]. Therefore it appears that minor Fe:Mn compositional changes in $\mathrm{Mn}_{5-x} \mathrm{Fe}_{x} \mathrm{Si}_{3}$ alloys can yield major changes in magnetic configuration, which might in turn explain (at least partly) the observed scatter in the literature about the magnetic anisotropy scheme for $\mathrm{MnFe}_{4} \mathrm{Si}_{3}$.

\section{Conclusions}

Both the magnetocaloric properties and magnetic anisotropy of $\mathrm{MnFe}_{4} \mathrm{Si}_{3}$ single crystal grown by flux method were investigated. By analyzing the field dependence of the magnetocaloric effect, the ferromagnetic transition at $T_{\mathrm{C}} \sim 323 \mathrm{~K}$ is found to be of second-order, which is fully compatible with the absence of thermal or magnetic hysteresis related to the transition. A relatively strong magnetic anisotropy for a transition-metals alloy is observed in $\mathrm{MnFe}_{4} \mathrm{Si}_{3}$ single crystal with the $c$ axis being a hard magnetic direction and an easy plane perpendicular to $c$. The anisotropy constants are determined over a broad temperature range, leading to a relatively large negative $K_{1}$. In contrast to former reports, X-ray diffraction experiments on field oriented polycrystalline materials confirm the present magnetic anisotropy scheme, indicating that the crystalline form or the single crystal preparation method is not the primary origin for the differences existing in the literature on $\mathrm{MnFe}_{4} \mathrm{Si}_{3}$.

\section{CRediT authorship contribution statement}

H. Yibole: Conceptualization, Investigation. W. Hanggai: Investigation. Z.Q. Ou: Investigation. R. Hamane: Investigation. V. Hardy: Investigation. F. Guillou: Investigation.

\section{Declaration of Competing Interest}

The authors declare that they have no known competing financial interests or personal relationships that could have appeared to influence the work reported in this paper.

\section{Acknowledgments}

The work is supported by Inner Mongolia Normal University (grant Nos. 2018YJRC002 and 2018YJRC003) and the Natural Science Foundation of China (grant Nos. 51850410514 for F.G.; 51961033, 11904188 for H.Y.; 11864031 for Z.Q.O.).

\section{Appendix A. Supplementary data}

Supplementary data to this article can be found online at https://doi. org/10.1016/j.jmmm.2020.166597.

\section{References}

[1] Y. Lecocq, P. Lecocq, M. Michel, C.R. Acad, Sci. Paris 258 (1964) 5655.

[2] C.E. Johnson, J.B. Forsyth, G.H. Lander, P.J. Brown, J. Appl. Phys. 39 (1968) 465, doi:10.1063/1.2163482

[3] K.S.V.L. Narasimhan, W.M. Reiff, H. Steinfink, R.L. Collins, J. Phys. Chem. Solids 31 (1970) 1511-1524, doi:10.1016/0022-3697(70)90035-1.

[4] G. Kappel, G. Fischer, A. Jaegle, Phys. Status Solid A 34 (1976) 691, doi:10.1002/ pssa.2210340233.

[5] D. Songlin, O. Tegus, E. Brück, J.C.P. Klaasse, F.R. de Boer, K.H.J. Buschow, J. Alloys Compds. 334 (2002) 249-252, doi:10.1016/S092-8388(01)01776-5.

[6] A. Candini, O. Moze, W. Kockelmann, J.M. Cadogan, E. Brück, O. Tegus, J. Appl. Phys. 95 (2004) 6819, doi:10.1063/1.1688219.

[7] O. Gourdon, M. Gottschlich, J. Persson, C. de la Cruz, V. Petricek, M.A. McGuire, T. Brückel, J. Solid State Chem. 216 (2014) 56-64, doi:10.1016/ j.jssc.2014.05.001.

[8] P. Hering, K. Friese, J. Voigt, J. Person, N. Aliouane, A. Grzechnik, A. Senshyn, T. Brückel, Chem. Mater. 27 (2015) 7128, doi:10.1021/acs.chemmater.5b03123.

[9] M. Herlitschke, B. Klobes, I. Sergueev, P. Hering, J. Perßon, R.P. Hermann, Phys Rev B 93 (2016), doi:10.1103/PhysRevB.93.094304 094304.

[10] N. Biniskos, S. Raymond, K. Schmalzl, A. Schneidewind, J. Voigt, R. Georgii, P. Hering, J. Persson, K. Friese, T. Brückel, Phys Rev B 96 (2017) 104407.

[11] R. Bouachraoui, Y. Ziat, Y. Sbai, O.E. Rhazouani, F. Goumrhar, L. Bahmad, J. Alloys and Compnds. 809 (2019), doi:10.1016/j.jallcom.2019.151785 151785

[12] K.A. Gschneidner Jr., Y. Mudryk, V.K. Pecharsky, Scr. Mater. 67 (2012) 572-577, doi:10.1016/j.scriptamat.2011.12.042.

[13] M. Werwiński, S. Kontos, K. Gunnarsson, P. Svedlindh, J. Cerdervall, V. Höglin, M. Sahlberg, A. Edström, O. Eriksson, J. Rusz, Phys. Rev. B 93 (2016), doi:10.1103/PhysRevB.93.174412 174412.

[14] T.N. Lamichhane, V. Taufour, S. Thimmaiah, D.S. Parker, S.L. Bud'ko, P.C. Canfield, J. Mag. Mag. Mater. 401 (2016) 525, doi:10.1016/j.jmmm.2015.10.088.

[15] B.T. Lejeune, B. Barua, I.J. McDonald, A.M. Gabay, L.H. Lewis, G.C. Hadjipanayis, J. Alloys Compds. 731 (2018) 995, doi:10.1016/j.jallcom.2017.10.061.

[16] F. Guillou, A.K. Pathak, D. Paudyal, Y. Mudryk, F. Wilhelm, A. Rogalev, V.K. Pecharsky, Nat. Commun. 9 (2018) 2925, doi:10.1038/s41467-018-05268-4

[17] V. Franco, J.S. Blazquez, A. Conde, Appl. Phys. Lett. 89 (2006), doi:10.1063/ 1.2399361222512.

[18] J.Y. Law, V. Franco, L.M. Moreno-Ramírez, A. Conde, D.Y. Karpenkov, I. Radulov, K.P. Skokov, O. Gutfleish, Nat. Commun. 9 (2018) 2680, doi:10.1038/ s41467-018-05111-w.

[19] K.A. Gschneidner Jr., V.K. Pecharsky, A.O. Tsokol, Rep. Prog. Phys. 68 (2005) 1479, doi:10.1088/0034-4885/68/6/R04.

[20] V.K. Pecharsky, K.A. Gschneidner Jr., A.O. Pecharsky, A.M. Tishin, Thermodynamics of the magnetocaloric effect, Phys. Rev. B 64 (2001), doi:10.1103/ PhysRevB.64.144406 144406.

[21] W. Sucksmith, F.R.S. Thompson, J.E. Thompson, Proc. Royal Soc. A 225 (1954) 362, doi:10.1098/rspa.1954.0209.

[22] M.B. Lyakhova, O.V. Zhdanova, Metal Sci. Heat Treat. 58 (2017) 587, doi:10.1007/s11041-017-0060-x. 
[23] A.S. Bolyachkin, D.S. Neznakhin, M.I. Bartashevich, J. Appl. Phys. 118 (2015), doi:10.1063/1.4936604 213902.

[24] A.S. Bolyachkin, D.S. Neznakhin, T.V. Garaeva, A.V. Andreev, M.I. Bartashevich, J. Mag. Mag. Mater. 426 (2017) 740, doi:10.1016/j.jmmm.2016.10.133.

[25] K.H.J. Buschow, F.R. de Boer, Physics of Magnetism and Magnetic Materials, Kluwer Academic Publishers, 2004. 